\title{
The Properties of Isobars in Shear Concentration Convective Flows of a Viscous Incompressible Fluid in a Plane Horizontal Layer with Motionless Boundaries
}

\author{
N. V. Burmasheva, ${ }^{1,2)}$ and E. Yu. Prosviryakov ${ }^{1,2, b)}$ \\ ${ }^{1}$ Institute of Engineering Science, Ural Branch of the Russian Academy of Sciences, \\ 34 Komsomolskaya St., Ekaterinburg, 620049, Russian Federation \\ ${ }^{2}$ Ural Federal University named after the first President of Russia B.N. Yeltsin, \\ 19 Mira St., Ekaterinburg, 620002, Russian Federation

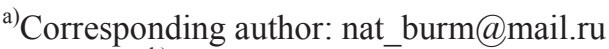 \\ b)evgen_pros@mail.ru
}

\begin{abstract}
It is known that convection contributes to the natural mixing of melts due to the uneven distribution of their density. This mixing can be enhanced by various devices and by changing the conditions surrounding the flow region of the medium, e.g. by changing the pressure at the boundaries of the flow region of such fluid media. A pressure difference inside the region filled with a viscous fluid can induce its flow. A classic example of such a flow is the Poiseuille flow. Moreover, if the pressure distribution depends also on horizontal coordinates (i.e., there is a pressure difference not only in the vertical direction), then longitudinal pressure gradients also generate additional flows, which are superimposed on the classical flow. The paper studies the effect of pressure on the shear convective flow of a viscous incompressible binary fluid in a horizontal layer. To describe such flows, we use a system of equations of concentration convection, which includes the equation of motion of a viscous binary fluid, the equation of a concentration change, and the incompressibility equation. The solution of the system of constitutive equations is sought with the use of the class of generalized solutions, in which the velocities depend only on the vertical coordinate, the pressure and concentration being also linearly dependent on the longitudinal (horizontal) coordinates. As the boundary conditions, it is assumed that the no-slip condition is met at the lower impermeable boundary, that the upper boundary of the layer is motionless, and that the distribution of salinity and pressure is specified on it. The solution of the formulated boundary value problem is a set of polynomial functions. The highest-degree polynomial describes background pressure. The study of the properties of background pressure and the longitudinal pressure gradients is in the focus of attention. It is shown that background pressure decreases strictly monotonically with moving away from the lower boundary of the layer, regardless of the control parameters of the boundary value problem. In this case, the longitudinal pressure gradients are also described by strictly monotonic functions, but the nature of the monotonicity is determined by the values of the longitudinal concentration gradients specified at the upper boundary of the fluid layer. The relevant findings are illustrated.
\end{abstract}

\section{BOUNDARY VALUE PROBLEM FORMULATION}

The flow of a viscous fluid in a horizontal layer of a given thickness $h$ is considered. The flow is induced by the uneven distribution of impurity (salinity) concentration in the fluid. In the case that the flow under study is a steadystate layered flow, the equations of the model describing these flows take the following form $[1,2]$ :

$$
\begin{gathered}
(\boldsymbol{V} \cdot \nabla) \boldsymbol{V}=-\nabla P+v \Delta \boldsymbol{V}+g \beta C \boldsymbol{k} ; \\
(\boldsymbol{V} \cdot \nabla) C=d \Delta C ; \\
\nabla \cdot \boldsymbol{V}=0 .
\end{gathered}
$$


Here, $V=\left(V_{x}(x, y, z), V_{y}(x, y, z), 0\right)$ is the velocity vector $V ; P(x, y, z)$ is deviation of pressure from hydrostatic, divided by the average density; $C(x, y, z)$ is the concentration of the light phase of a binary mixture; $v$ and $d$ are the kinematic (molecular) viscosity of the fluid and the diffusion coefficient, respectively; $g$ is the acceleration of gravity; $\nabla$ and $\Delta$ are the Hamilton and Laplace three-dimensional operators, respectively.

The nonlinear system of partial differential equations (1) consists of the Navier-Stokes equation in the Boussinesq approximation, the incompressibility equation, and the equation for changing the concentration of the light phase of a binary mixture. Moreover, system (1) includes five scalar equations with respect to four unknown functions (velocities $V_{x}$ and $V y$, concentration $C$, and pressure $P$ ). This means that system (1) is overdetermined. The balance between the number of equations and the number of unknowns is fulfilled when the "redundant" equations are satisfied. Note that, if a solution to system (1) is sought in the class [3-7]

$$
\begin{gathered}
V_{x}=U(z) ; V_{y}=V(z) ; V_{z}=0 ; \\
P=P_{0}(z)+x P_{1}(z)+y P_{2}(z) ; C=C_{0}(z)+x C_{1}(z)+y C_{2}(z),
\end{gathered}
$$

the incompressibility equation is satisfied identically. Moreover, by substituting the class (2)-(3) into system (1), this system is reduced to an equivalent system of ordinary differential equations with respect to decomposition components in (2)-(3):

$$
\begin{gathered}
C_{1}{ }^{\prime}=0 ; C_{2}{ }^{\prime}=0 ; P_{1}{ }^{\prime}=g \beta C_{1} ; P_{2}{ }^{\prime}=g \beta C_{2} ; \\
v V^{\prime \prime}=P_{2}, v U^{\prime \prime}=P_{1}, d C_{0}{ }^{\prime \prime}=U C_{1}+V C_{2}, P_{0}{ }^{\prime}=g \beta C_{0} .
\end{gathered}
$$

The prime in system (4) denotes differentiation with respect to the vertical (transverse) coordinate $z$. The equations of system (4) are integrated according to the given order.

To determine the integration constants appearing in the solution of the equations of system (4), we consider the following boundary value problem. Assume that the solid boundary $\mathrm{z}=0$ of the fluid layer is impermeable and that the fluid no-slip condition is fulfilled on it [8-10],

$$
\left.V_{x}\right|_{z=0}=\left.V_{y}\right|_{z=0}=0,\left.\frac{\partial C}{\partial \boldsymbol{n}}\right|_{z=0}=0,
$$

where $\boldsymbol{n}$ is the normal to the lower boundary of the layer.

Assume that the pressure distribution at the upper fixed boundary $z=h$ is homogeneous and that the impurity concentration distribution linearly depends on the longitudinal (horizontal) coordinates,

$$
\left.V_{x}\right|_{z=h}=\left.V_{y}\right|_{z=h}=0,\left.P\right|_{z=h}=S_{0},\left.C\right|_{z=h}=a x+b y .
$$

\section{EQUATION SYSTEM SOLUTION}

The solution of the boundary value problem (4)-(6) is polynomial and written as follows:

$$
\begin{gathered}
U=\psi Z(-2+Z)(-1+Z) ; \\
V=\gamma Z(-2+Z)(-1+Z) ; \\
C_{1}=a ; C_{2}=b ; \\
C_{0}=\lambda(-1+Z)\left(8+8 Z+8 Z^{2}-12 Z^{3}+3 Z^{4}\right) ; \\
P_{1}=\xi_{1}(-1+Z) ; P_{2}=\xi_{2}(-1+Z) ;
\end{gathered}
$$




$$
P_{0}=S_{0}+\xi_{3}(-1+Z)^{2}\left(11+6 Z+Z^{2}-4 Z^{3}+Z^{4}\right) .
$$

Here,

$$
\begin{gathered}
Z=z / h, \psi=a g \beta h^{3} /(6 v), \gamma=b g \beta h^{3} /(6 v), \lambda=\left(a^{2}+b^{2}\right) g h^{5} \beta /(360 d v), \\
\xi_{1}=a g \beta h, \xi_{2}=b g \beta h, \xi_{3}=\left(a^{2}+b^{2}\right) g^{2} h^{6} \beta^{2} /(720 d v) .
\end{gathered}
$$

In what follows, the pressure field distribution is analyzed in detail.

\section{PRESSURE FIELD INVESTIGATION}

It can be seen from Eq. (7) that the longitudinal (horizontal) gradients $P_{1}, P_{2}$ of the pressure field $P$ are strictly monotonic functions taking only the values of the same sign. The sign of these components is determined by the sign of the longitudinal gradients $a, b$ of the concentration field $C$,

$$
P_{i}^{\prime}=\xi_{i}(i=1,2) .
$$

The properties of the background pressure $P_{0}$ are determined, in particular, by the properties of the polynomial

$$
f(Z)=(-1+Z)^{2}\left(11+6 Z+Z^{2}-4 Z^{3}+Z^{4}\right) .
$$

The function $f$ is a monotonically decreasing non-negative function (Fig. 1).

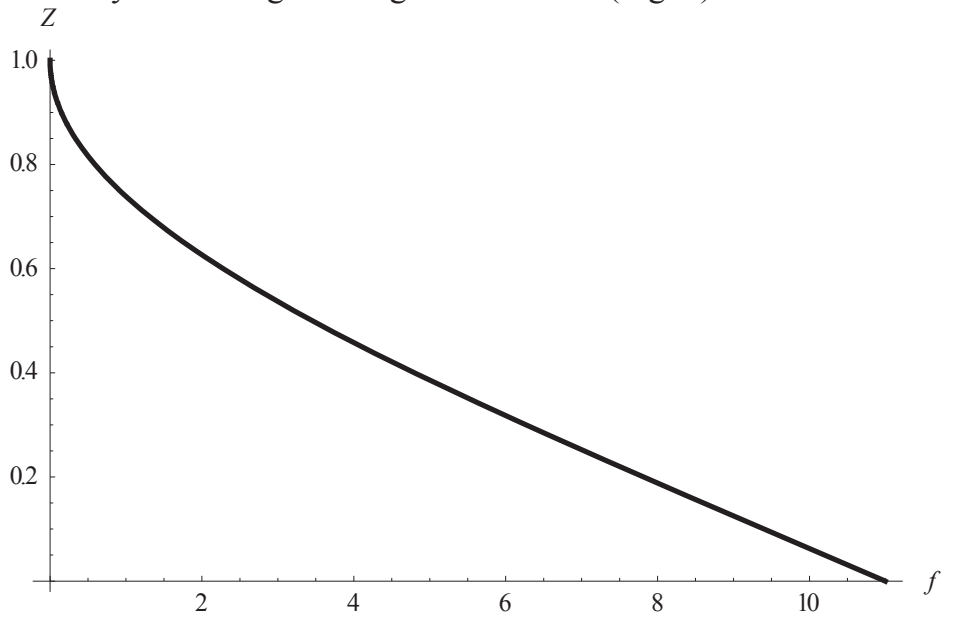

FIGURE 1. The behavior of the function $f(Z)$

Since the coefficient $\xi_{3}$ is non-negative, the term $\xi_{3} f$ included in the expression for background pressure is also a monotonically decreasing function taking only non-negative values. This means that the pressure field $P_{0}$ can have zero values only when the coefficient $S_{0}$ is negative. Note that this option is possible since, in fact, pressure $P$ is here considered as deviation of pressure from hydrostatic, normalized to fluid density. This deviation can be both positive and negative. Due to the monotonicity of the polynomial $f$, we can easily write the parameter conditions at which the background pressure can stratify [11-13] into two zones,

$$
S_{0}\left(S_{0}+11 \xi_{3}\right)<0
$$

As an example, let us further consider the case when the condition represented by Eq. (8) is met, which means the presence of a stratification point of the field $P_{0}$ (Fig. 2). 


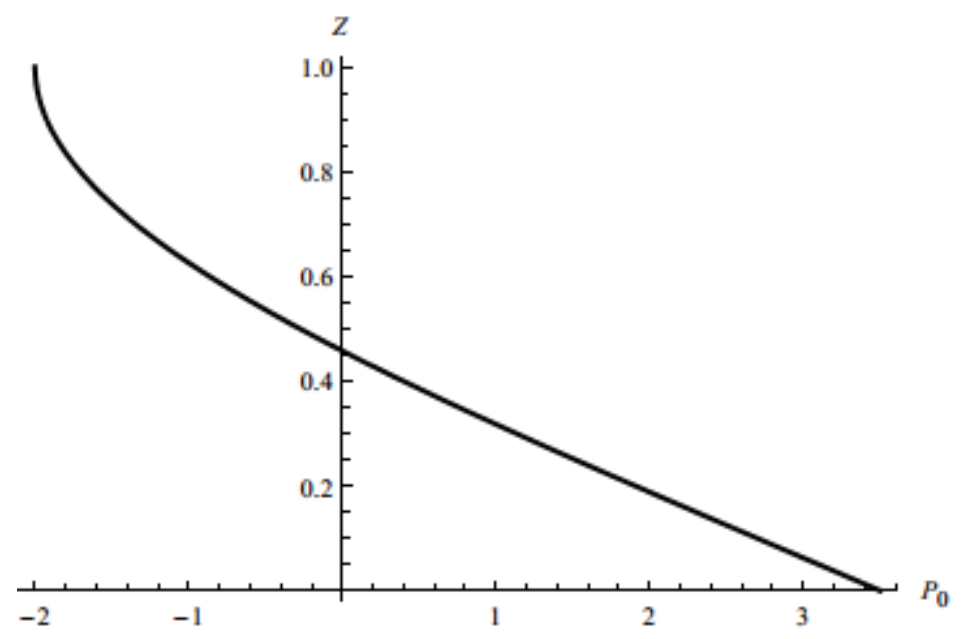

FIGURE 2. The profile of the pressure $P_{0}\left(S_{0}=-2, \xi_{3}=0.5\right)$

The shape of the isolines of the resulting pressure field $P$ depends on the values of the coefficients $S_{0}$ and $\xi_{\mathrm{i}}$ (Figs. 3 and 4).

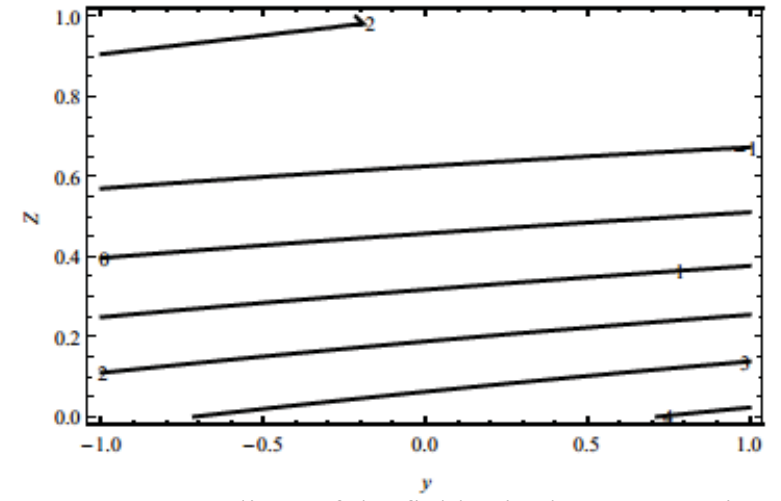

FIGURE 3. Isolines of the field $P$ in the cross section

$$
\left(S_{0}=-2, \xi_{3}=0.5, \xi_{2}=-0.7\right)
$$

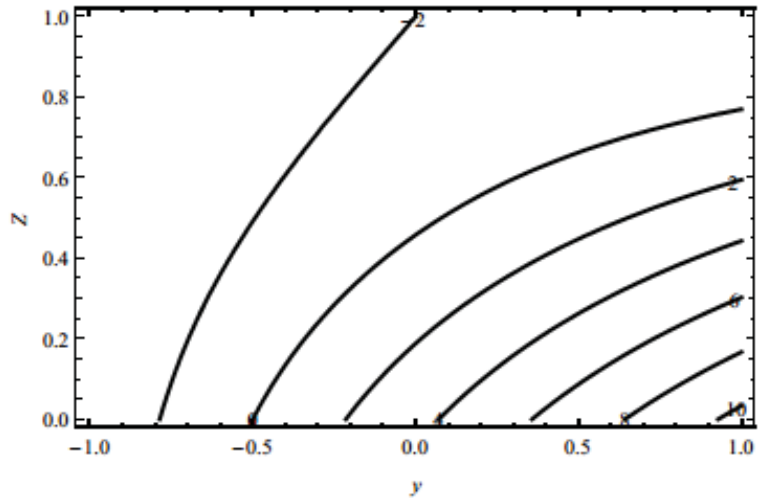

FIGURE 4. Isolines of the field $P$ in the cross section $x=0$

$\left(S_{0}=-2, \xi_{3}=0.5, \xi_{2}=-7\right)$

\section{CONCLUSION}

The obtained exact solution for the concentration convection of a viscous incompressible fluid in a horizontal layer is able to describe the stratification of the pressure field. It has been demonstrated that background pressure can be stratified into no more than two zones.

\section{REFERENCES}

1. G. Z. Gershuni, E. M. Zhukhovitskii, Convective Stability of Incompressible Fluids (Wiley/Keter Press, Jerusalem, 1976).

2. D. D. Joseph, Stability of Fluid Motions (Springer-Verlag, Berlin, Heidelberg, New York, 1976).

3. M .Couette, Ann. Chim. Phys. (Ser. 6) 21, 433-510 (1890).

4. A. V. Gorshkov and E. Yu. Prosviryakov, AIP Conf. Proc. 2053, 040029-1-040029-7 (2018).

5. N. V. Burmasheva, E. A. Larina, and E. Yu. Prosviryakov, AIP Conference Proceedings 2176, 030023 (2019).

6. V. V. Privalova and E. Yu. Prosviryakov, Vestn. Samar. Gos. Tekhn. Univ., Ser. Fiz.-Mat. Nauki 22(3), $532-$ 548 (2018). 
7. N. S. Knutova and K. G. Shvarts, Fluid Dyn. 50(3), 340-350 (2015).

8. D. V. Knyazev, Journal of Applied Mechanics and Technical Physics 52(2), 212-217 (2011).

9. L. Prandtl, O. Tietjens, Funadamentals of Hydro- and Aeromechanic (McGraw-Hill Book Company, New York - London, 1934).

10. S. V. Goldstein, Modern Developments in Fluid Mechanics (Oxford Univ. Press, Oxford, 1938).

11. V. N. Zyryanov and A. P. Frolov, Water Resources 33(1), 1-9 (2006).

12. J. M. Dorrepaal, Journal of Fluid Mechanics 163(1), 141-147 (1986).

13. V. Shtern, Counterflows. Paradoxical Fluid Mechanics Phenomena (Cambridge University Press, Cambridge 2012). 